\title{
Satellite Cloud Image Retrieval With Grid Based Inscribed Circle Method
}

\author{
Shiming Xia ${ }^{1, a}$, Qian $\mathrm{Li}^{2, b^{*}}$ and Jing Zhang ${ }^{3, \mathrm{c}}$ \\ ${ }^{1,3}$ College of Meteorology and Oceanography, PLA University of Science and Technology, Nanjing, \\ 211101, China \\ ${ }^{2}$ State Full address of second author, including country Key Laboratory for Novel Software \\ Technology, Nanjing University, Nanjing 210093, China \\ a email: 15295753714@163.com, ${ }^{b^{*}}$ email: public_liqian@163.com, ${ }^{c}$ email: 1052744845@qq.com
}

Keywords: Satellite cloud image; Feature vector; Similarity retrieval; Rotation invariance; Scale invariance

\begin{abstract}
Satellite cloud image retrieval can help the forecasters positioning similar weather in the past quickly and improve the reliability of the weather prediction. This article attempts to use the Zernike moment and Grid based method to extract the region-based features of cloud images. First of all, carry out the pretreatment of separating cloud from background, extract effective features and generate the features library of the cloud image library. Then calculate the similarity between a given cloud image and the cloud library. Finally, output the final results according to the order of similarity. After comparing the retrieval results of the two methods, we put forward an improved algorithm named grid based inscribed circle method(GBIC). Experiments show that this method possesses the advantages of the above two methods and can retrieve cloud images with similar visual features from the history cloud image library.
\end{abstract}

\section{Introduction}

Satellite cloud images play an important role in business domain of weather. As the satellite images possess high time and space resolution, and can be used to track cloud formation, the relationship between weather system strengthen and precipitation continuously. So satellite images help the forecasters to improve the accuracy of the weather forecast [1]. At present, numerous satellite cloud images are accumulated in the database and failed to get effective usage [2].We can adopt the image retrieval methods on historical satellite cloud images for similarity retrieval. So that we can draw lessons from the past weather condition to improve the accuracy of the weather prediction for the future. After analysis, we find that the meteorological satellite cloud images show a greater degree of differentiation in shape features. Therefore, we adapt the shape features for similarity retrieval on satellite cloud images.

In a satellite cloud image, the shape features of the cloud refers to the different strength of the target light reflection, or its radiation emission of different distribution patterns of light and shade degree of the image point [3]. The shape of the cloud structure helps to understand the kinds of cloud and the cloud formation process. For a satellite cloud image, as the completely extraction of the outline is difficult, and cloud information used by contour-based features is much less than region-based features. And region-based features regard all pixels as a whole, thus region-based features are robust to noise and shape change[4]. So we only adopt the region-based features for similarity retrieval.

\section{Framework}

To take advantage of shape region-based features on the cloud images retrieval, we proposed the system framework as shown in Fig.1.The framework consists of two parts, offline generation of feature library and online retrieval. During the phase of offline generation, first of all, carry out the pretreatment of separation on the cloud images. Namely adopt histogram equalization process to improve cloud images' contrast and carry out half threshold method processing [5] (pixels higher than the threshold value are unchanged, below the threshold value become zero pixels). Then further 
extract the shape-based features of the separated cloud images, and combined with the description of corresponding cloud image files for the online retrieval. During the online retrieval phase, the user inputs a cloud image for retrieval firstly. Carry out the pretreatment of separation on the given image and further extract the feature. Then calculate the distance between the given image and the characteristic library. At last ,output the retrieval results according to the sorted order based on the vector distance.

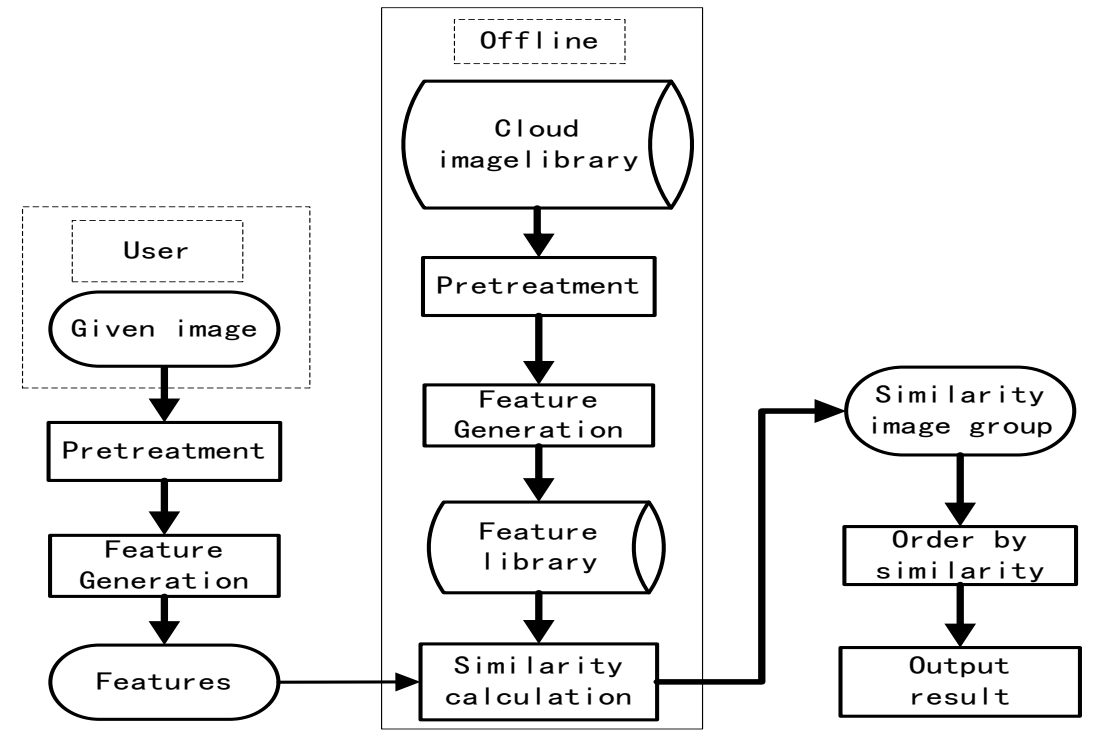

Fig.1: The system framework

Region-based features. Region-based features are mainly includes the area of the region, Euler number, dispersion ratio, eccentricity, geometric moment, Zernike moment, Legendre moments, grid method, and the radius of angle transformation, etc. In this paper, we try to use Zernike moment and grid method to carry out similarity retrieval on the satellite cloud images.

Zernike moment. Zernike moment is based on the Zernike polynomial orthogonal function [6], the set of orthogonal polynomial used is complete inside a standard circle (the radius is 1 ). When computing a Zernike moment of a given image, define the image gravity (also known as the gravity center) as the center of the circle [7], the pixel coordinates are mapped to the standard circle. Zernike moment is a plural moment and the modulus value of Zernike moment is generally regarded as a feature to describe the object shape, the Zernike moment is defined as [8]

$$
Z_{n m}=\frac{n+1}{\pi} \sum_{x} \sum_{y} V_{n m}^{*}(x, y) f(x, y), x^{2}+y^{2} \leq 1
$$

Where, $V_{n m}^{*}(x, y)=V_{n m}(\rho \cos \theta, \rho \sin \theta)=R_{n m}(\rho) \exp (j m \theta)$, and $R_{n m}(\rho)$ is defined as

$$
R_{n m}(\rho)=\sum_{s=0}^{(n-|m|) / 2}(-1)^{s} \frac{(n-s) !}{s !\left(\frac{n+|m|}{2}-s\right) !\left(\frac{n-|m|}{2}-s\right) !} \times \rho^{n-2 s}
$$

Where, $n$ and $m$ are nonnegative integers, also make sure $n-|m|$ is even and $n \geq|m|$.Choosing different $n$ and $m$ that meet the conditions can form a feature vector.

Grid method. The grid shape descriptor is proposed by Lu [9]. Basically, divide a given cloud image into a certain number of neat grid cells, in this article it is divided into $8 \times 8$ grid cells, as shown in Fig.2. A grid of cells is overlaid on a cloud image, the grid is then scanned from left to right and top to bottom. Calculate the average pixel value of each grid cell $\mu_{i}$ and the global average value $\mu$, when $\mu_{i}$ is greater than the global average $\mu$, the grid cell is assigned as 1 , otherwise 0 , so each cloud image corresponding to a 64 bits binary string. The advantages are its simplicity and possessing the scale invariance. The main problem is lack of rotation invariance. Scale invariance is refers to the mage scale variation does not affect the feature, as long as the image can still be divided into $8 \times 8$ grid 
cells. The same picture, the grid feature values usually change a lot after rotating 90 degree, so the grid method lack of rotation invariance.

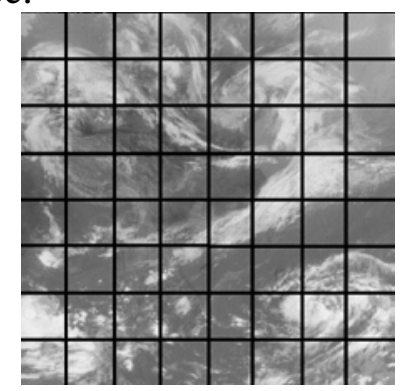

Fig.2 $8 \times 8$ grid cells

By the experiment of section 5.2, we can find that Zernike moment is better in terms of rotation invariance, but the retrieval result is not ideal. While the retrieval result of grid method is better, this method is better in terms of scale invariance, but not in the rotation invariance. During the experiment, we try several methods to combine Zernike moment and grid method, but the combined retrieval result is poor, therefore we propose the grid based inscribed circle (GBIC) method. Here, we only extract characteristic inside the inscribed circle part of a cloud image. In order to possess the rotation invariance, we extract grid characteristic of the inscribed circle part of cloud image every 30 degree angle, thus each cloud image corresponds 12 directions grid features. During the similarity comparison, for a given cloud image, first of all, extract its grid features; then compare this grid feature with the 12 directions grid values in the characteristic library and find out the most similar direction; at last, calculate the similarity degree between the two images. Fig.3 (a) is an inscribed circle, Fig.3 (b) is the inscribed circle part of a cloud image, Fig.3 (c) is the meshing of an inscribed circle.

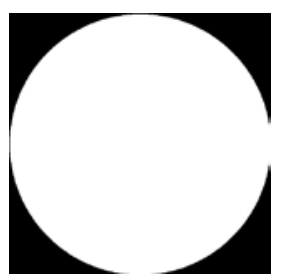

(a) inscribed circle

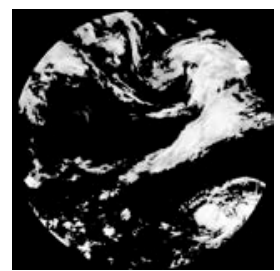

(b) inscribed circle cloud image

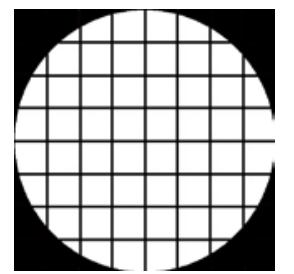

(c) the meshing of inscribed circle

Fig.3: grid inscribed circle

We can find that for a cloud library, if calculate the corresponding 12 directions grid features of each cloud image in advance, it will obviously make the workload of off-line feature library generation become at least 12 times as much as the grid method. In fact, we needn't to generate grid features of all the 12 directions, we only need to generate the grid characteristic of the 0 degree direction, but during the similarity retrieval, it needs to generate 12 directions grid features of the given cloud image for retrieval, then use the 12 grid features respectively compare with the 0 degree grid characteristic of each image in the cloud library, find out the most similar grid characteristic as the similarity degree of the two images. By doing so, it can greatly reduce the pressure of off-line feature library generation, while online searching pressure increase very little(because for only one cloud image, the time difference between generating 12 directions features and generating one direction characteristic is very narrow, which can be neglected). In this way, we can achieve a better retrieval effect, and will not spend too much time.

Distance measurement. During online retrieval, we need to set a reasonable distance metric criterion to reflect the similarity of two different features vector. Calculated the distance between a given cloud image and feature vector in the feature library based on the $\chi^{2}$ distance. The $\chi^{2}$ distance [10] is defined as

$$
d_{\chi^{2}}(A, B)=\sum_{i=1}^{n} \frac{\left(a_{i}-m_{i}\right)^{2}}{m_{i}}
$$

Where, $A$ 、 $B$ are features of two n-dimensional vectors, $m_{i}$ is defined as 


$$
m_{i}=\frac{a_{i}+b_{i}}{2}
$$

Where, $a_{i}$ and $b_{i}$ corresponding with the $i$ component of $A$ 、 $B$ eigenvectors.

By the definition of $\chi^{2}$ distance, we can see that it can reduce the influence of dimension and the features of each component needn't be normalized.

\section{Results and analysis}

To test the above methods, we choose a personal computer whose main frequency is $2.26 \mathrm{GHz}$ and memory is 2 GB. We adopted Net3.0 and OpenCV as the programming environment. The experimental data is received from satellite named Situation 2 in year 2010 and 2011. During the comparison of the shape features, we only adapt 900 infrared cloud images under lambert projection received in year 2011 for experiments, the experiments were mainly carried out to validate the rotation invariance and the scale invariance. During the experiment for rotation invariance, we rotated the 900 images for 180 degrees, then put the rotated images into the cloud library, so the total number of the cloud library is 1800. During the experiment for the scale invariance, the same 900 images will be reduced to a quarter and then put the reduced images into the cloud library, so we can get another cloud library of 1800 images.

Pretreatment results. During the cloud image pretreatment phase, we carry out the pretreatment of separation on the infrared satellite cloud image named "EILY0630. AWX", which was received on May 6, 2011 at 3. The processing results are shown in Fig. 4. Fig.4 (a) is the original cloud image, Fig.4 (b) is the cloud image after histogram equalization, Fig.4 (c) is the result of cloud separation. The threshold value is 160 , we can see that using this method can get better separation of cloud from the ground background.

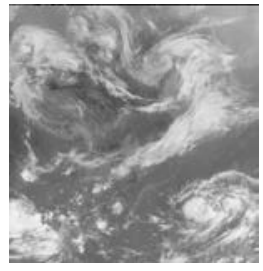

(a)original image

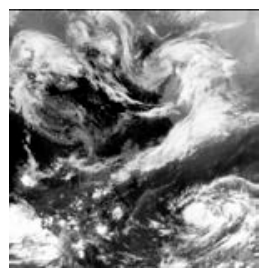

(b) histogram

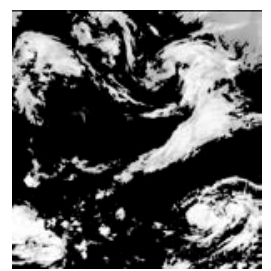

(c) cloud separation

equalization

Fig. 4 pretreatment of cloud image

Retrieval results and analysis. During the similarity retrieval of satellite cloud images, we use the infrared satellite cloud image on May 6, 2011 at 3 named "EILY0630. AWX", and respectively adopt Zernike moment, grid method and grid based inscribed circle method to carry out similarity retrieval on infrared cloud library received in year 2011.

Results and analysis of Zernike moment. The first 16 most similar results are shown in Fig.5 by the method of Zernike moment, the retrieval results are listed according to the similarity degree from high to low. The marks under each figure respectively represent the time of cloud image and the similarity degree between a given image and the retrieval result. In order to distinguish the original cloud image from the rotated image, we add "rotated" behind the similarity degree of rotated image. In order to distinguish the original image from the image reduced to a quarter, we add "reduced" behind the similarity degree of reduced image. From Fig. 5 (a), a cloud image and the rotated image can be detected at the same time, which indicates that Zernike moment has very good rotation invariance. However, the reliability of the retrieval is poor, some of the retrieval results in visual similarity degree is not high enough. Looking at Fig.5 (b), the reduced cloud images can't be retrieved, suggesting that Zernike moment does not have scale invariance. 


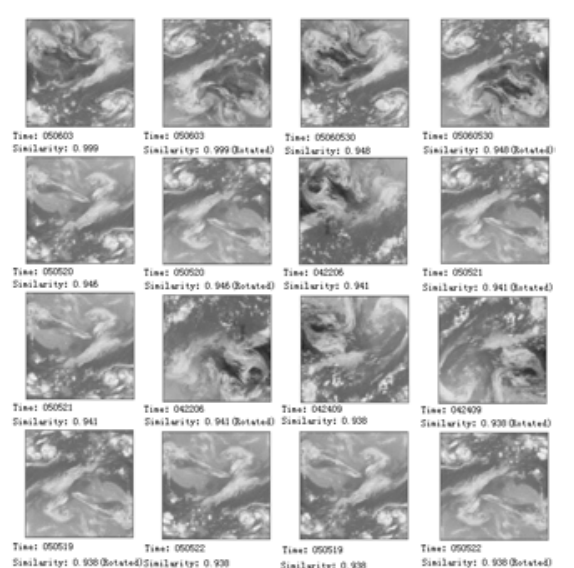

(a)Rotation invariance validation

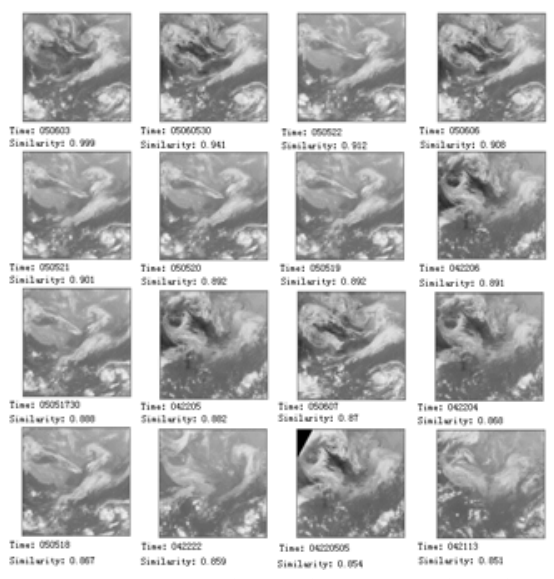

(b) scale invariance validation

Fig.5: Zernike moment related experiments

Results and analysis of grid method. The first 16 of the most similar results are shown in Fig.6. From Fig.6 (a), we can see that none rotated image been detected, so the grid method does not have rotation invariance, but the reliability of the retrieval is better, the time of the retrieval results is very close to the time of the given cloud image for retrieval, which conforms to the continuous movement of cloud within a time scope. Looking at Fig.6 (b), the cloud image and the reduced cloud image can be retrieved at the same time, suggesting that grid method has scale invariance.

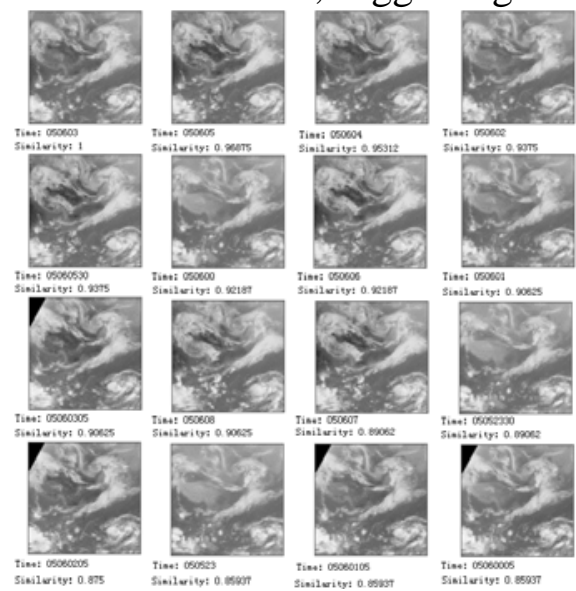

(a)rotation invariance validation

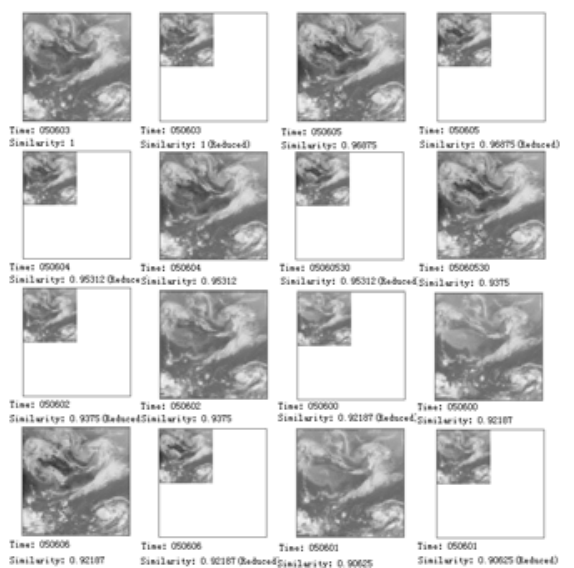

(b) scale invariance validation

Fig.6: relevant experimental grid method

Results and analysis of GBIC method. The first 16 of the most similar search results are shown in Fig.7 by the GBIC method. From Fig.7 (a), the GBIC method has rotation invariance, and the retrieval reliability is better. The Fig.7 (b) shows that the method has the scale invariance.

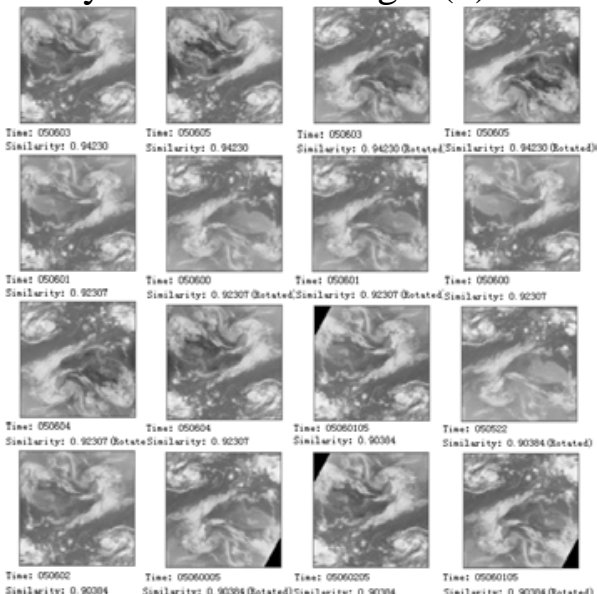

(a)rotation invariance validation

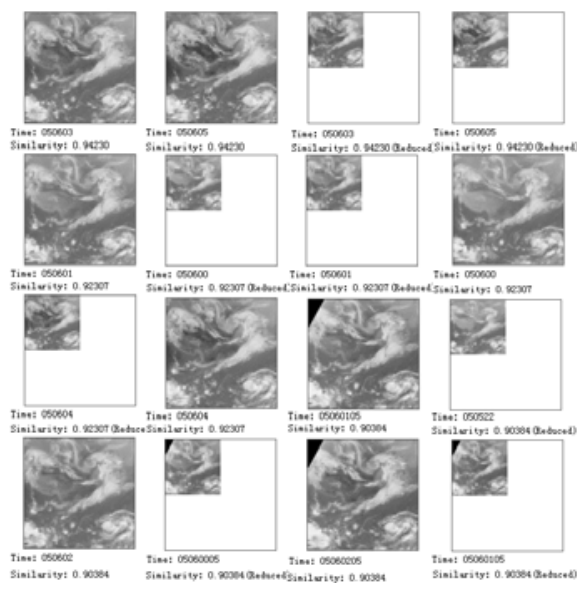

(b) scale invariance validation

Fig.7:GBIC method related experiments 


\section{Summary}

In this article, there are several points worth noticing. Firstly, during the cloud image pretreatment phase, it is difficult to find a universal threshold value to carry out the cloud separation. However, if we first carry out the histogram equalization progressing, we can choose a value between 150 and 170 as the universal threshold value. Secondly, by comparing the advantages and disadvantages of several region-based methods, we propose an improved method. The experimental results show that the method can extract the characteristic information of the cloud effectively, and can work well for similarity retrieval on historical satellite cloud library, so this method has a certain application prospect. In the future work, we will focus on how to make fusion of shape features and texture features to improve the reliability of retrieval.

\section{References}

[1] Sun Xuejin, Wang Xiaolei, Li Hao, Atmospheric sounding learning. Beijing: meteorological press,2009.

[2] Peng Hong, Li Qiong, Wen Quanche. Key techniques of content-based image retrieva. Microcomputer information ,2007, 23 (1-3) 7-10.

[3] Chen Weimin, Satellite meteorology. Beijing: meteorological press,2005,pp.1-50.

[4] Sun Junding, Zhao Shan, Low-level feature extraction and image retrieval technology. Beijing: electronic industry press, 2009.

[5] Li Junshan, Li Xuhui, Digital image processing. Beijing: tsinghua university press, 2010.

[6] Wang Shuizhang, Based on the texture image retrieval technology research. Graduate student dissertation of Taiyuan university of technology,2008,pp.140-150.

[7] Yang Zhengwu, Fang Tao, Based on the Zernike moment image normalization technique research . Computer engineering,2004,30 (12), pp. 1-3.

[8] Li Jinquan, Wang Jianwei, Chen Shanben, Wu Lin. An improved orthogonal Zernike moment subpixel edge detection algorithm. Optical technology,2003,29(4), pp. 500-503.

[9] Lu G.J., Sajjanhar A., Region-based shape representation and similarity measure suitable for the content-based image retrieval. Multimedia System,1999,7 (2),pp. 165-174.

[10] Zhang D.S., Lu G.J., Evaluation of similarity measurement for image retrieval, IEEE Int. Conf. Neural Networks and Signal Processing, Nan Jing, China, 2003, pp.928-931. 